\title{
Evaluation of Digital Drawing Tests and Paper-and-Pencil Drawing Tests for the Screening of Mild Cognitive Impairment and Dementia: A Systematic Review and Meta-analysis of Diagnostic Studies
}

\author{
Joyce Y. C. Chan ${ }^{1}$ - Baker K. K. Bat ${ }^{2}$. Adrian Wong ${ }^{1} \cdot$ Tak Kit Chan $^{3} \cdot$ Zhaohua Huo $^{3} \cdot$ Benjamin H. K. Yip ${ }^{3}$. \\ Timothy C. Y. Kowk ${ }^{1} \cdot$ Kelvin K. F. Tsoi ${ }^{2,3}$
}

Received: 19 March 2021 / Accepted: 9 August 2021 / Published online: 16 October 2021

(c) The Author(s) 2021

\begin{abstract}
Digital drawing tests have been proposed for cognitive screening over the past decade. However, the diagnostic performance is still to clarify. The objective of this study was to evaluate the diagnostic performance among different types of digital and paper-and-pencil drawing tests in the screening of mild cognitive impairment (MCI) and dementia. Diagnostic studies evaluating digital or paper-and-pencil drawing tests for the screening of MCI or dementia were identified from OVID databases, included Embase, MEDLINE, CINAHL, and PsycINFO. Studies evaluated any type of drawing tests for the screening of MCI or dementia and compared with healthy controls. This study was performed according to PRISMA and the guidelines proposed by the Cochrane Diagnostic Test Accuracy Working Group. A bivariate random-effects model was used to compare the diagnostic performance of these drawing tests and presented with a summary receiver-operating characteristic curve. The primary outcome was the diagnostic performance of clock drawing test (CDT). Other types of drawing tests were the secondary outcomes. A total of 90 studies with 22,567 participants were included. In the screening of MCI, the pooled sensitivity and specificity of the digital CDT was $0.86(95 \% \mathrm{CI}=0.75$ to 0.92$)$ and 0.92 (95\% CI $=0.69$ to 0.98$)$, respectively. For the paper-and-pencil CDT, the pooled sensitivity and specificity of brief scoring method was $0.63(95 \% \mathrm{CI}=0.49$ to 0.75$)$ and 0.77 ( $95 \% \mathrm{CI}=0.68$ to 0.84 ), and detailed scoring method was 0.63 (95\% $\mathrm{CI}=0.56$ to 0.71$)$ and $0.72(95 \% \mathrm{CI}=0.65$ to 0.78 ). In the screening of dementia, the pooled sensitivity and specificity of the digital CDT was 0.83 (95\% CI $=0.72$ to 0.90$)$ and 0.87 (95\% CI $=0.79$ to 0.92 ). The performances of the digital and paper-and-pencil pentagon drawing tests were comparable in the screening of dementia. The digital CDT demonstrated better diagnostic performance than paper-and-pencil CDT for MCI. Other types of digital drawing tests showed comparable performance with paper-and-pencil formats. Therefore, digital drawing tests can be used as an alternative tool for the screening of $\mathrm{MCI}$ and dementia.
\end{abstract}

Keywords Digital drawing test $\cdot$ Digital CDT $\cdot$ MCI $\cdot$ Dementia

\section{Background}

There is a growing concern globally for mild cognitive impairment (MCI) and dementia due to the increasing aging population. Dementia is a syndrome in which there is

Kelvin K. F. Tsoi

kelvintsoi@cuhk.edu.hk

1 Department of Medicine and Therapeutics, The Chinese University of Hong Kong, Hong Kong, China

2 Stanley Ho Big Data Decision Analytics Research Centre, The Chinese University of Hong Kong, Hong Kong, China

3 JC School of Public Health and Primary Care, The Chinese University of Hong Kong, Hong Kong, China progressive deterioration of cognitive function such as memory, visuospatial abilities, executive functions, and thinking (World Health Organization, 2019). MCI is recognized as the intermediate stage between normal aging and dementia (Petersen et al., 1999; Winblad et al., 2004). Early detection of MCI and dementia by cognitive screening tests can help patients and their family members to receive timely proper dementia-related care and support from health care professionals (Prince et al., 2011)

Drawing tests are quick, easy to use, cognitive screening tests that are commonly used for the screening of MCI and dementia. Drawing tests can be applied easily and can assess different neuropsychological functions, such as visuospatial ability and executive functions (Aprahamian 
et al., 2009; Ehreke et al., 2010). Deterioration of visuospatial abilities and executive functions are common cognitive symptoms in patients with MCI and dementia (Pal et al., 2016; Traykov et al., 2007). There are different types of drawing tests such as the clock drawing test (CDT) (Shulman et al., 1986; Sunderland et al., 1989), pentagon drawing test (Cormack et al., 2004), and cube drawing test (Ota et al., 2015). These tests can be either used alone or combined in a multi-domain cognitive test, such as Montreal Cognitive Assessment (MoCA) (Nasreddine et al., 2005). The CDT is the most extensively used drawing test (Aprahamian et al., 2009; Ehreke et al., 2010). In the early development of the CDT, it was mainly used to screen visuospatial and visual-constructional disorders associated with lesions in the parietal region of the brain, such as post-stroke dementia (Aprahamian et al., 2009). The usage of CDT is widened nowadays, and the CDT is widely used for the screening of MCI and dementia (Tsoi et al., 2015). There are different scoring methods of the CDT such as the 6-point scoring method (Shulman et al., 1986) and the 10-point scoring method (Sunderland et al., 1989). Studies indicate that the CDT is a good test for the screening of patients with dementia (Aprahamian et al., 2009; Park et al., 2018), but that MCI patient performance is fair and may overlook degenerative processes (Breton et al., 2019; Ehreke et al., 2010; Pinto \& Peters, 2009; Tsoi et al., 2017).

Due to the weakness of paper-and-pencil drawing tests and the advancement of technology, digital drawing tests have evolved over the past decade. Digital drawing tests can record and assess drawing characteristics such as total time spent, contour, and drawing methods, which can be considered when discriminating between MCI and dementia (Heymann et al., 2018). Studies showed that on-air movements can enhance the sensitivity of identifying patients with MCI (Garre-Olmo et al., 2017; Müller et al., 2017). The pressure applied when drawing can be another indicator to discriminate elders with MCI and healthy aging (Faundez-Zanuy et al., 2013).

A meta-analysis found that the diagnostic performance of digital cognitive tests and paper-and-pencil cognitive tests are comparable (Chan et al., 2018). However, previous studies seldom compare the diagnostic performance of digital drawing tests and paper-and-pencil drawing tests specifically. Therefore, the objective of this study was to evaluate the diagnostic performance of different types of digital drawing tests and paperin-pencil drawing tests for the screening of $\mathrm{MCI}$ and dementia.

\section{Methods}

The current study was performed according to the standard guidelines for the systematic review of diagnostic studies, including the Preferred Reporting Items for Systematic
Reviews and Meta-Analyses (PRISMA) (Moher et al., 2009) and the guidelines proposed by the Cochrane Diagnostic Test Accuracy Working Group (Leeflang et al., 2008; Macaskill et al., 2010). This study is registered as CRD42020166750 in PROSPERO.

\section{Search Strategy}

Literature searches were performed in OVID databases, included Embase, MEDLINE, CINAHL, and PsycINFO. Keywords included "cognitive impairment", "MCI", "dementia", "draw test", "digital draw", "Clock drawing test", "Pentagon test", "Cube draw", "Tree draw", "House draw' and "Rey-Osterrieth", "ROCF", "Spiral" and "infinity loops" (Supplementary Table 1). The search duration was from the earliest available dates in each database to the $31 \mathrm{st}$ of March 2020. Diagnostic studies comparing the accuracy of the drawing tests for MCI and dementia were identified from the title and abstract preview of all search records. Literature searches were also extended to the Digital Dissertation Consortium database and WorldCat for identification of unpublished theses or grey literature. Manual searches were extended to the bibliographies of the review articles and studies that were included in this meta-analysis. No language restriction was adopted.

\section{Inclusion and Exclusion Criteria}

Studies were included if they met the following inclusion criteria:

1. The study used any type of drawing tests for the screening of MCI or dementia;

2. The study recruited participants with MCI or dementia in any clinical or community settings and compared them with cognitively healthy controls;

3. Participants with MCI or dementia were confirmed with standardized diagnostic criteria, including the Petersen criterion (Petersen et al., 1999), the report of International Working Group on Mild Cognitive Impairment (Winblad et al., 2004), the recommendations of the National Institute on Aging and the Alzheimer's Association (Albert et al., 2011), the National Institute of Neurological and Communicative Disorders and Stroke and the Alzheimer's Disease and Related Disorders Association (NINCDS-ADRDA) (McKhann et al., 1984), any version of the Diagnostic and Statistical Manual of Mental Disorder (DSM) (American Psychiatric Association, 2013), consensus by qualified clinicians using the Clinical Dementia Rating (CDR) (Morris, 1993) or standardized neuropsychological tests. Studies reported 
cases of very mild dementia, questionable dementia or cognitive impairments with no dementia (CIND), were further studied to confirm whether they included participants with MCI;

4. The diagnostic performance of the drawing tests was summarized in terms of sensitivity and specificity, or data that could be used to derive those values were provided.

Studies were excluded if a study only evaluated different types of MCI or dementia, such as to identify patients with Parkinson's disease dementia from Alzheimer's disease.

\section{Data Extraction}

Two investigators (TKC, BKK) independently evaluated the relevance of search results and extracted the data into an Excel spreadsheet. The spreadsheet was used to record the demographic details of included articles, such as the year of publication, the study location, the number of MCI or dementia participants and controls, the mean age of participants, the percentage of male participants, and the diagnostic criteria and cutoff values used to define patients with MCI or dementia. We also recorded the sensitivity and specificity, or true-positive, false-positive, true-negative, and false-negative values, of each drawing test for result analysis. When a study presented different cutoff values to show the performance of a test, only the result from a recommended cutoff value in the article was chosen. When a study recommended more than one cutoff value, the cutoff value presented in the abstract of the article was selected. When there were discrepancies in the study eligibility or data extraction, the third investigator (JYC) would make the definitive decision. The Cohen's kappa was used to evaluate the inter-rater variability.

\section{Type of Drawing Tests}

The digital drawing tests included the digital CDT, digital pentagon drawing test, digital Rey-Osterrieth complex figure (ROCF), digital tree drawing test, digital house drawing test, and digital spiral test. The paper-and-pencil drawing tests included the CDT, pentagon drawing test, cube drawing test, and ROCF. We further categorized paper-and-pencil CDT into the brief scoring method (i.e. $\leq 9$ score), and the detailed scoring method (i.e. $>9$ score).

\section{Risk of Bias and Reporting Quality}

The Quality Assessment of Diagnostic Accuracy Studies 2 (QUADAS-2) instrument (Whiting et al., 2011) was used to evaluate the potential risks of bias (ROB). The assessment areas included, 1. selection of patient, 2. execution of the screening tests, 3 . execution of the reference standard, and 4. presentation on the patient flow and timing to have the reference standard and index tests. The methodology section of the STARD statement (Standards for Reporting of Diagnostic Accuracy) (Bossuyt et al., 2003) was used to evaluate the study quality. An 8-point scale was designed to evaluate the study quality, which included: 1 . a clear definition on study population, 2 . adequate details of recruitment of participants, 3 . description of sampling of participant selection, 4. description of data collection plan, 5. description of reference standard and its rationale, 6. specifications of the drawing tests, 7. rationales for cutoff values, and 8 . methods of calculation of diagnostic performance.

\section{Outcomes}

The primary outcome of this study was the diagnostic performance of the CDT for the screening of MCI and dementia. The secondary outcome was the diagnostic performance of other types of drawing tests.

\section{Data Synthesis and Statistical Analysis}

A bivariate random-effects model was used to combine the overall sensitivity and specificity of each drawing test (Reitsma et al., 2005). Forest plots were used as the graphical presentation for the pooled sensitivity and specificity. A diagnostic odds ratio was used as a single indicator of the test performance across different thresholds of cutoff values (Glas et al., 2003). A hierarchical summary receiveroperating characteristic (HSROC) curve was generated to present the summary estimates of sensitivity and specificity along with corresponding 95\% confidence interval (95\% CI) and prediction region (Rutter \& Gatsonis, 2001). The area under the HSROC curve (AUC) was calculated. The approach of DerSimonian and Laird was applied when Hessian matrix was unstable (DerSimonian \& Laird, 1986). Statistical heterogeneity among the trials was assessed by $\mathrm{I}^{2}$. The diagnostic performances of digital and paper-andpencil drawing tests were compared by meta-regression models, with $P<0.05$ indicates a statistically significant difference. Publication bias was conducted by a regression of diagnostic log odds ratio against $1 /$ sqrt (effective sample size), weighting by effective sample size, with $P<0.10$ for the slope coefficient indicating significant asymmetry (Deeks et al., 2005). Sensitivity analysis was conducted according to the scoring methods of paper-and-pencil clock drawing tests. The statistical analyses were performed with the Midas procedures in STATA, version 11 (StataCorp) and meta-disc version 1.4. 


\section{Results}

\section{Literature Search and Study Selection}

A total of 7,180 abstracts were identified in OVID databases, 37 papers were identified from the bibliography, and 363 abstracts were identified from WorldCat. After excluding the irrelevant or duplication of titles, 502 articles were further evaluated. A total of 412 articles were excluded (Cohen's Kappa statistics at $85 \%$ between the investigators) due to the following reasons: 36 studies were systematic review or meta-analysis, 335 studies were not studied the diagnostic performance of a drawing test, 24 abstracts or presentation posters were lack of diagnostic result, 5 studies were not recruited patients with $\mathrm{MCI}$ or dementia, and 12 studies were not recruited participants with normal cognition as a control group. As a result, a total of 90 studies were eligible for this systematic review and meta-analysis (Fig. 1).

\section{Study Characteristics}

A total of 90 studies with 2,810 participants with MCI, 7,751 participants with dementia, and 12,006 controls were included in this systematic review and metaanalysis. The mean age of the participants ranged from 58 to 85 (Supplementary Table 2). The percentage of male participants ranged from 4 to $99 \%$. Seventy-six studies recruited participants with MCI or dementia in an out-patient clinic or the community, whereas all other studies recruited participants in hospital or an old age home. Six studies used digital CDT, while other digital drawing tests included pentagon drawing test $(\mathrm{n}=2)$, tree drawing test $(n=2)$, ROCF $(n=1)$, house drawing test $(n=1)$, and spiral drawing test $(n=1)$. Paper-and-pencil CDT was the most commonly used drawing test, as 45 studies used the detailed scoring method (i.e. $>9$ score), and 35 studies used the brief scoring method (i.e. $\leq 9$ score). Other paper-andpencil drawing tests included the pentagon drawing test $(n=5)$, cube drawing test $(n=3)$ and ROCF $(n=1)$. In the assessment of ROB, nine studies were assessed as high risk of bias, including selection of patients $(n=6)$, execution of the index test $(n=1)$, execution of the reference standard $(\mathrm{n}=3)$ and flow and timing $(\mathrm{n}=4)($ Supplementary Table 3$)$.

\section{Performance of Digital and Paper-and-pencil CDT in the Screening of $\mathrm{MCl}$}

Four studies used the digital CDT for the screening of MCI, and a total of 179 participants with MCI and 467 controls were included. The sensitivities ranged from 0.67 to 0.94 and specificities ranged from 0.79 to 0.94 across individual studies. The heterogeneity with $\mathrm{I}^{2}$ statistic for sensitivity and specificity were 0.81 and 0.92 , respectively. The pooled sensitivity and specificity of four studies using digital CDT were 0.86 (95\% CI $=0.75$ to 0.92$)$ and $0.92(95 \% \mathrm{CI}=0.69$ to 0.98 ), respectively (Table 1a, Fig. 2, Supplementary

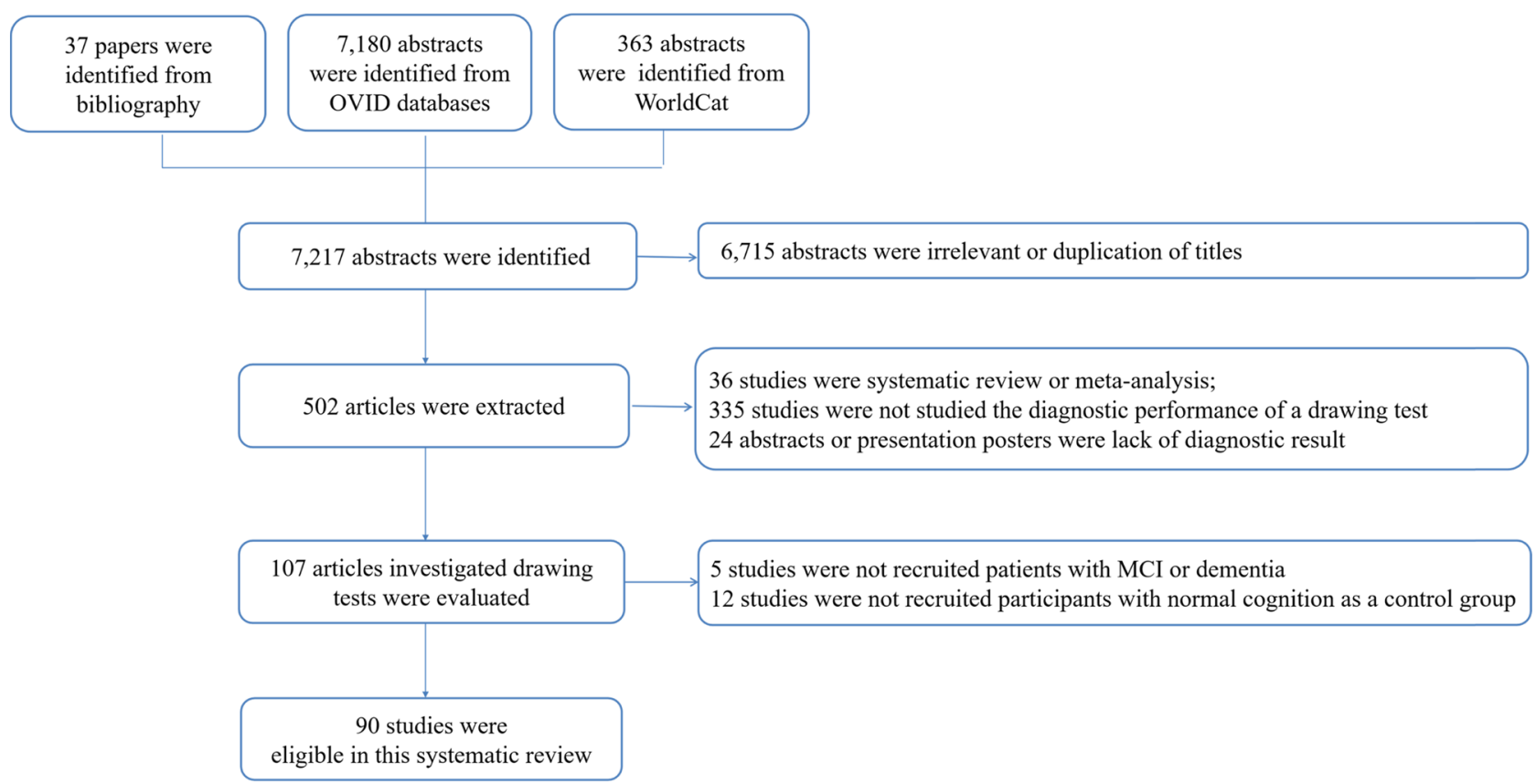

Fig. 1 Summary of Literature search 
Table 4). The pooled AUC was $87 \%$ (95\% $\mathrm{CI}=84 \%$ to $90 \%)$ (Supplementary Fig. 1). A non-significant $P$-value (0.42) for the slope coefficient suggested symmetry in the data and a low likelihood of publication bias (Supplementary Fig. 2). Nine studies used brief scoring method of paper-and-pencil CDT. The heterogeneity with $\mathrm{I}^{2}$ statistic for sensitivity and specificity were 0.92 and 0.93 , respectively. The pooled sensitivity and specificity were $0.63(95 \% \mathrm{CI}=0.49$ to 0.75$)$ and 0.77 (95\% CI $=0.68$ to 0.84$)$, respectively. The pooled AUC was $77 \%$ (95\% CI $=74 \%$ to $81 \%$ ). Twenty-one studies used detailed scoring method of paper-and-pencil CDT. The heterogeneity with $\mathrm{I}^{2}$ statistic for sensitivity and specificity were 0.87 and 0.86 , respectively. The pooled sensitivity and specificity were $0.63(95 \% \mathrm{CI}=0.56$ to 0.71$)$ and 0.72 ( $95 \% \mathrm{CI}=0.65$ to 0.78 ), respectively. The pooled AUC was $74 \%$ (95\% CI $=69 \%$ to $77 \%$ ). The diagnostic performance of digital CDT was significantly better than the brief scoring methods $(P=0.02)$ and detailed scoring methods $(P<0.001)$ of paper-and-pencil CDT in the meta-regression models.

\section{Performance of Digital and Paper-and-pencil CDT in the Screening of Dementia}

Six studies used the digital CDT for the screening of dementia, and a total of 505 participants with dementia and 915 controls were included. The sensitivities ranged from 0.63 to 0.94 and specificities ranged from 0.77 to 1.00 across individual studies. The heterogeneity with $\mathrm{I}^{2}$ statistic for sensitivity and specificity were 0.87 and 0.78 , respectively. The pooled sensitivity and specificity with bivariate random-effects model were $0.83(95 \% \mathrm{CI}=0.72$ to 0.90 ) and 0.87 (95\% CI $=0.79$ to 0.92 ) (Table $1 \mathrm{~b}$, Fig. 3). The pooled AUC was $92 \%$ (95\% CI $=89 \%$ to $94 \%$ ) (Supplementary Fig. 3). A non-significant $P$-value (0.93) for the slope coefficient suggested symmetry in the data and a low likelihood of publication bias. Thirty studies used brief scoring method of the CDT. The heterogeneity with $\mathrm{I}^{2}$ statistic for sensitivity and specificity were 0.86 and 0.83 , respectively. The pooled sensitivity and specificity were $0.83(95 \% \mathrm{CI}=0.77$ to 0.87$)$ and 0.80 ( $95 \% \mathrm{CI}=0.74$ to 0.85 ), respectively. The pooled AUC was $88 \%$ ( $95 \% \mathrm{CI}=85 \%$ to $91 \%$ ). Thirty-five studies used detailed scoring method of the CDT. The heterogeneity with $\mathrm{I}^{2}$ statistic for sensitivity and specificity were 0.82 and 0.93 , respectively. The pooled sensitivity and specificity were $0.80(95 \% \mathrm{CI}=0.76$ to 0.83$)$ and 0.81 (95\% $\mathrm{CI}=0.75$ to 0.86 ), respectively. The pooled AUC was $87 \%$ (95\% CI $=84 \%$ to $90 \%$ ). No significant difference was found between the diagnostic performance of digital CDT and brief scoring methods $(P=0.33)$ and detailed scoring methods $(P=0.35)$ of paper-and-pencil CDT in the meta-regression model.

Table 1 Sensitivity and Specificity of Digital and Paper-and-Pencil Clock Drawing Tests

\begin{tabular}{|c|c|c|c|c|c|c|c|}
\hline $\begin{array}{l}\text { Type of Clock } \\
\text { Drawing Tests }\end{array}$ & No. of study & $\begin{array}{l}\text { Sensitivity } \\
(95 \% \text { CI })\end{array}$ & $\begin{array}{l}\text { Specificity } \\
\text { (95\% CI) }\end{array}$ & $\begin{array}{l}\text { LR + } \\
(95 \% \text { CI })\end{array}$ & $\begin{array}{l}\text { LR- } \\
\text { (95\% CI) }\end{array}$ & $\begin{array}{l}\text { DOR } \\
(95 \% \text { CI })\end{array}$ & $\begin{array}{l}\text { AUC } \\
(95 \% \text { CI })\end{array}$ \\
\hline \multicolumn{8}{|l|}{ a. MCI } \\
\hline Digital CDT & 4 & $0.86(0.75-0.92)$ & $0.92(0.69-0.98)$ & $10.6(2.30-49.0)$ & $0.15(0.08-0.29)$ & $69.0(10.6-449)$ & $\begin{array}{l}87 \%(84 \%- \\
90 \%)\end{array}$ \\
\hline $\begin{array}{l}\text { Paper-and-pencil } \\
\text { CDT } \\
\text {-Brief Scoring }(\leq 9 \\
\text { points) }\end{array}$ & 9 & $0.63(0.49-0.75)$ & $0.77(0.68-0.84)$ & $2.74(1.94-3.88)$ & $0.48(0.34-0.68)$ & $5.71(3.06-10.7)$ & $\begin{array}{l}77 \%(74 \%- \\
81 \%)\end{array}$ \\
\hline $\begin{array}{l}\text { Paper-and-pencil } \\
\text { CDT } \\
\text {-Detailed Scoring } \\
\text { (>9 points) }\end{array}$ & 21 & $0.63(0.56-0.71)$ & $0.72(0.65-0.78)$ & $2.29(1.92-2.73)$ & $0.50(0.43-0.59)$ & $0.54(3.47-5.93)$ & $\begin{array}{l}74 \%(69 \%- \\
77 \%)\end{array}$ \\
\hline \multicolumn{8}{|l|}{ b. Dementia } \\
\hline Digital CDT & 6 & $0.83(0.72-0.90)$ & $0.87(0.79-0.92)$ & $6.35(3.9-10.5)$ & $0.20(0.12-0.33)$ & $32.2(13.7-75.9)$ & $\begin{array}{l}92 \%(89 \%- \\
94 \%)\end{array}$ \\
\hline $\begin{array}{l}\text { Paper-and-pencil } \\
\text { CDT } \\
\text {-Brief Scoring }(\leq 9 \\
\text { points) }\end{array}$ & 30 & $0.83(0.77-0.87)$ & $0.80(0.74-0.85)$ & $4.07(3.15-5.26)$ & $0.22(0.16-0.29)$ & $18.7(12.2-28.7)$ & $\begin{array}{l}88 \%(85 \%- \\
91 \%)\end{array}$ \\
\hline $\begin{array}{l}\text { Paper-and-pencil } \\
\text { CDT } \\
\text {-Detailed Scoring } \\
\text { (>9 points) }\end{array}$ & 35 & $0.80(0.76-0.83)$ & $0.81(0.75-0.86)$ & $4.24(3.24-5.54)$ & $0.25(0.21-0.30)$ & $17.1(12.0-24.5)$ & $\begin{array}{l}87 \%(84 \%- \\
90 \%)\end{array}$ \\
\hline
\end{tabular}

$C D T$ Clock Drawing Test, $M C I$ Mild Cognitive Impairment, $L R+$ Positive likelihood ratio, $L R$ - Negative likelihood ratio, $D O R$ Diagnostic odds ratio, AUC Area under the curve, $C I$ Confidence interval 
i) Digital clock drawing test
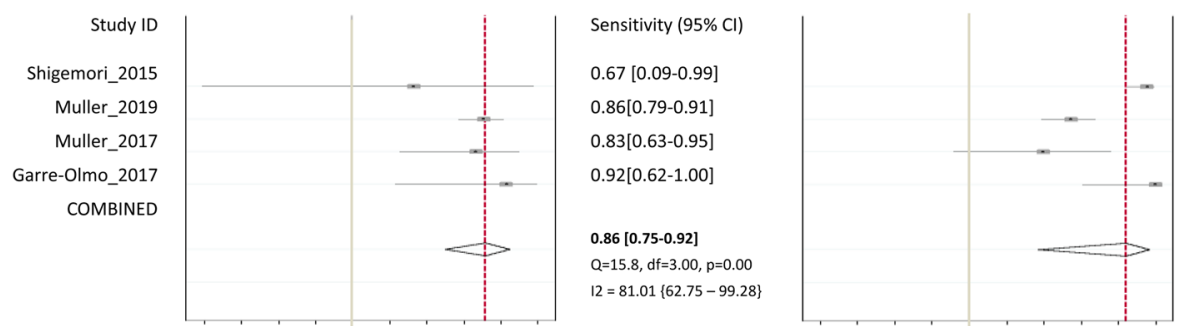

Specificity $(95 \% \mathrm{Cl})$

0.98[0.92-1.00]

$0.77[0.69-0.84]$

$0.70[0.46-0.88]$

$1.00[0.80-1.00]$

$0.92[0.69-0.98]$

$Q=37.15, d f=3.00, p=0.00$

$12=91.92\{85.69-98.16\}$

ii) Paper-and-Pencil Clock Drawing Test (Brief scoring methods)
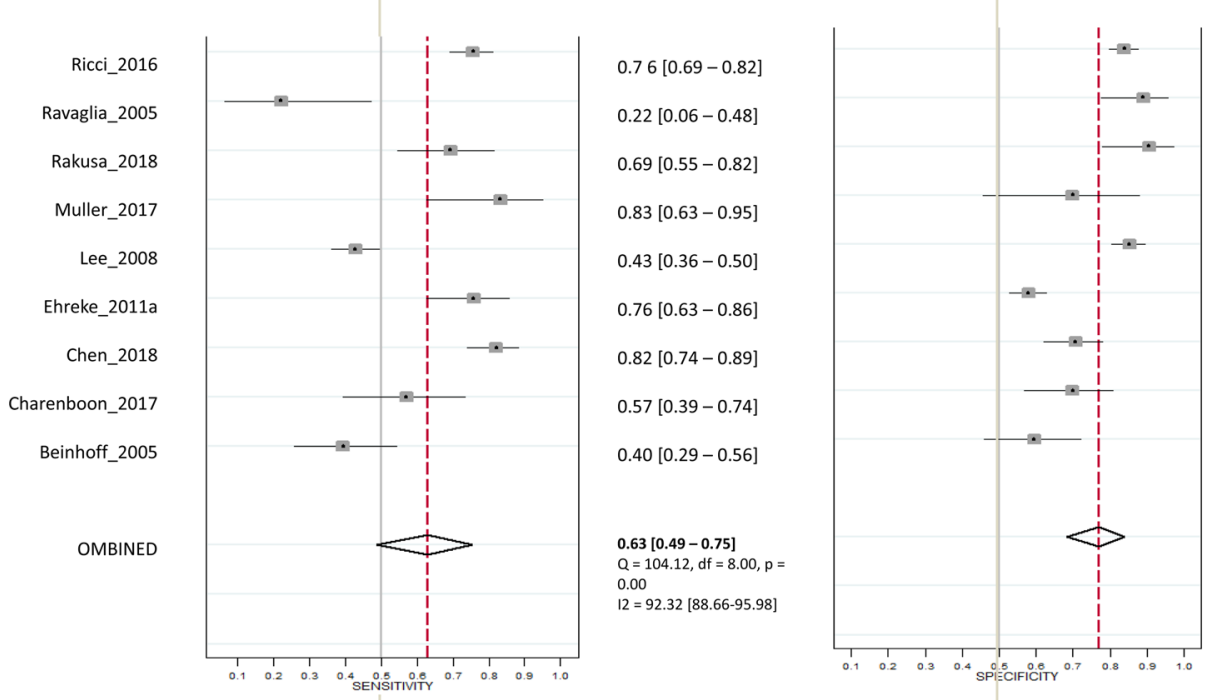

$0.84[0.80-0.88]$

$0.89[0.78-0.96]$

0.91 [0.78-0.97]

$0.70[0.46-0.88]$

$0.85[0.80-0.90]$

0.58 [0.53-0.63]

$0.71[0.62-0.78]$

$0.70[0.57-0.81]$

$0.60[0.45-0.72]$

$0.77[0.68-0.84]$

$Q=111.83, \mathrm{df}=8.00, p=0.00$

$12=92.85[88.51-96.18]$

i) Paper-and-Pencil Clock Drawing Test (Detailed scoring methods)
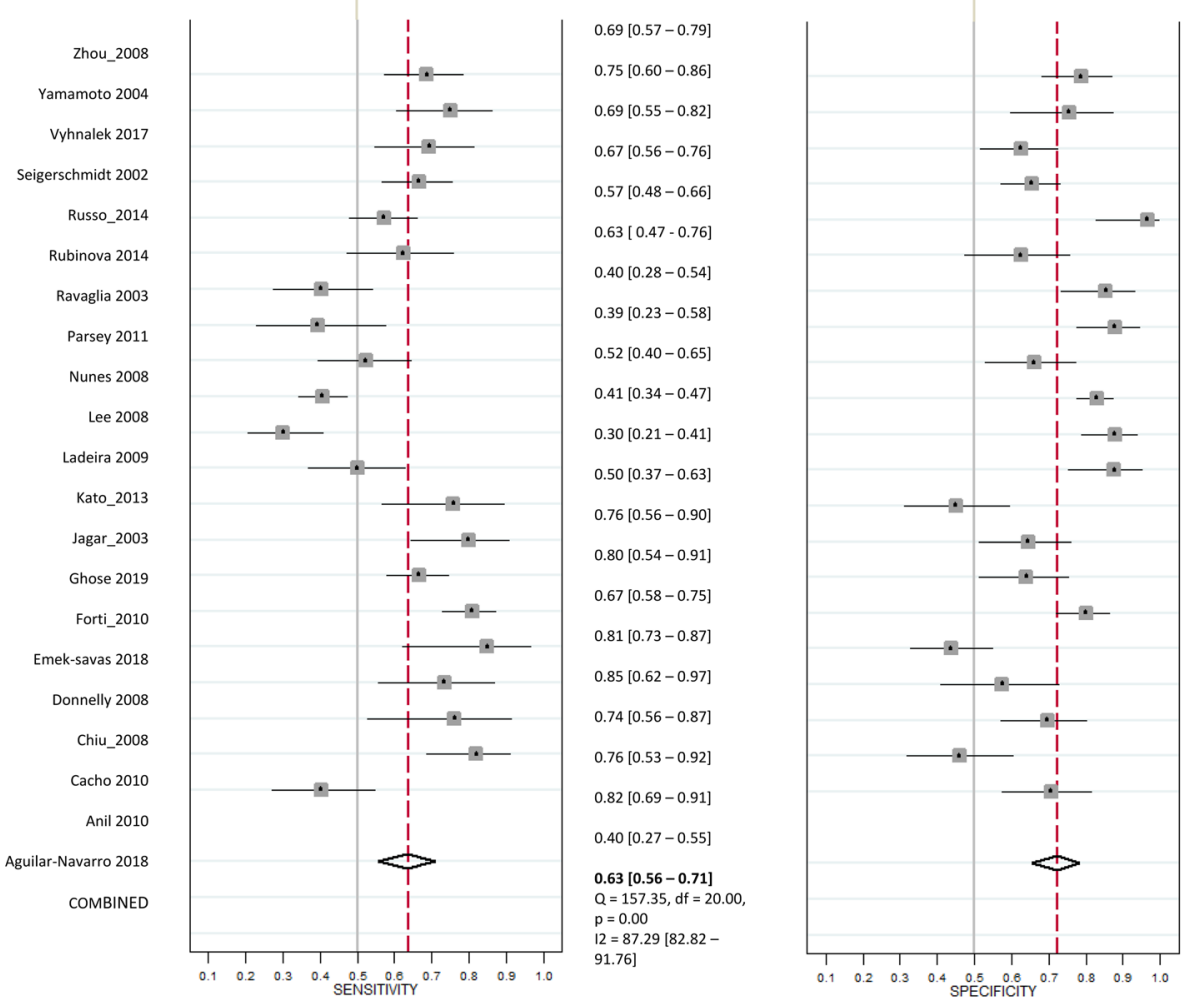

$0.79[0.68-0.87]$

$0.76[0.60-0.88]$

$0.63[0.52-0.73]$

$0.65[0.57-0.73]$

$0.97[0.83-1.00]$

$0.63[0.47-0.76]$

$0.85[0.73-0.94]$

$0.88[0.78-0.95]$

$0.66[0.53-0.78]$

0.83 [0.78-0.88]

$0.30[0.21-0.41]$

0.88 [0.75-0.95]

$0.49[0.31-0.60]$

$0.65[0.51-0.76]$

$0.64[0.51-0.76]$

$0.80[0.72-0.87]$

$0.44[0.33-0.55]$

$0.57[0.41-0.73]$

$0.70[0.57-0.80]$

$0.46[0.32-0.61]$

$0.71[0.57-0.82]$

0.72 [0.65-0.78]

$Q=144.54, d f=20.00, p=0.00$

$Q=144.54, d f=20.00, p=0.00$
$12=86.17[81.19-91.15]$

Fig. 2 Studies of Digital and Paper-and-Pencil Clock Drawing Tests for the Screening of MCI 
i) Digital clock drawing test

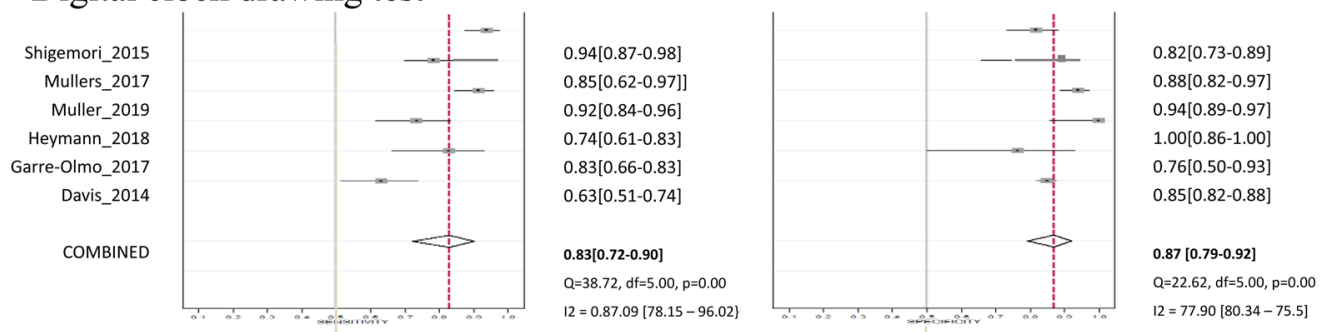

ii) Paper-and-Pencil Clock Drawing Test (Brief scoring methods)

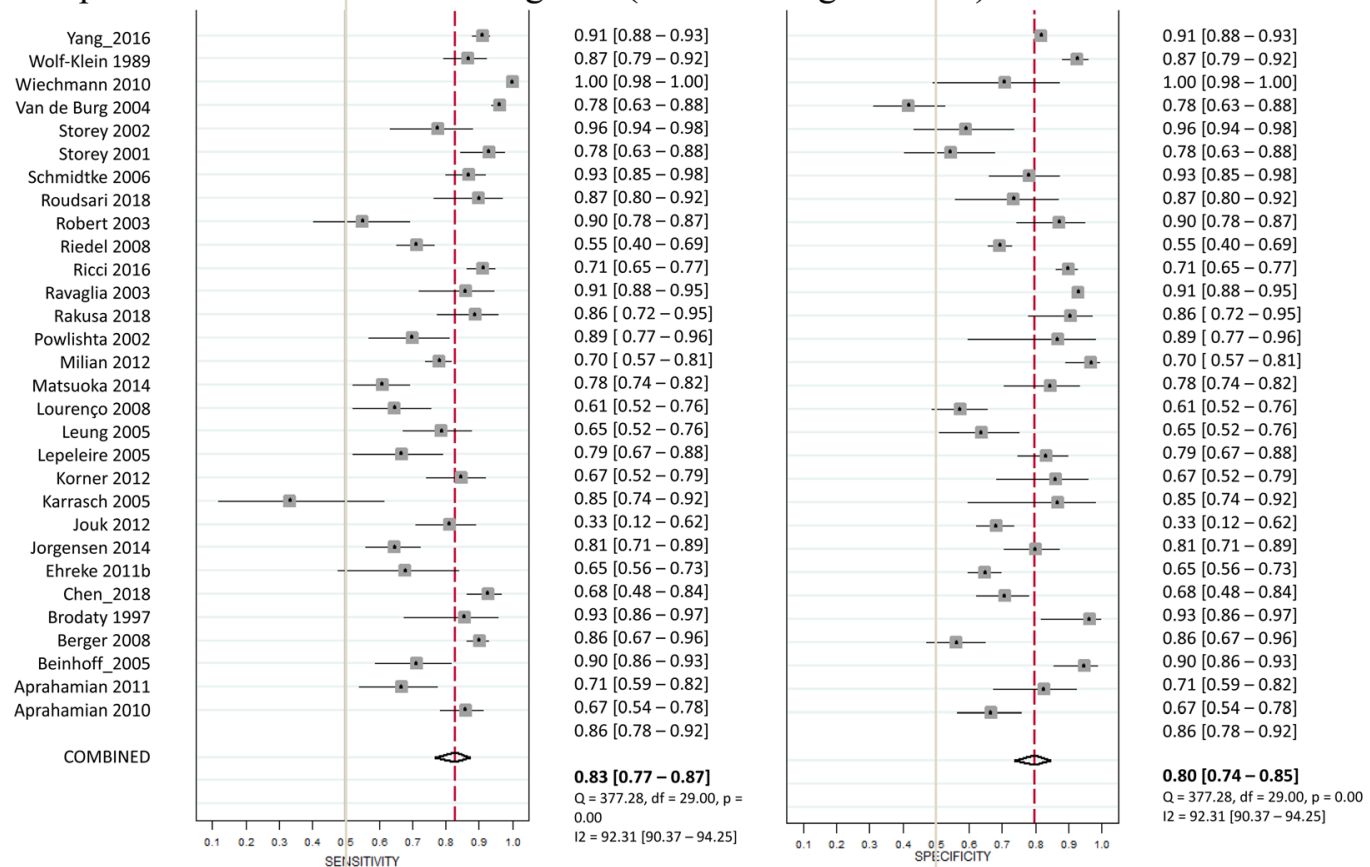

iii) Paper-and-Pencil Clock Drawing Test (Detailed scoring methods)

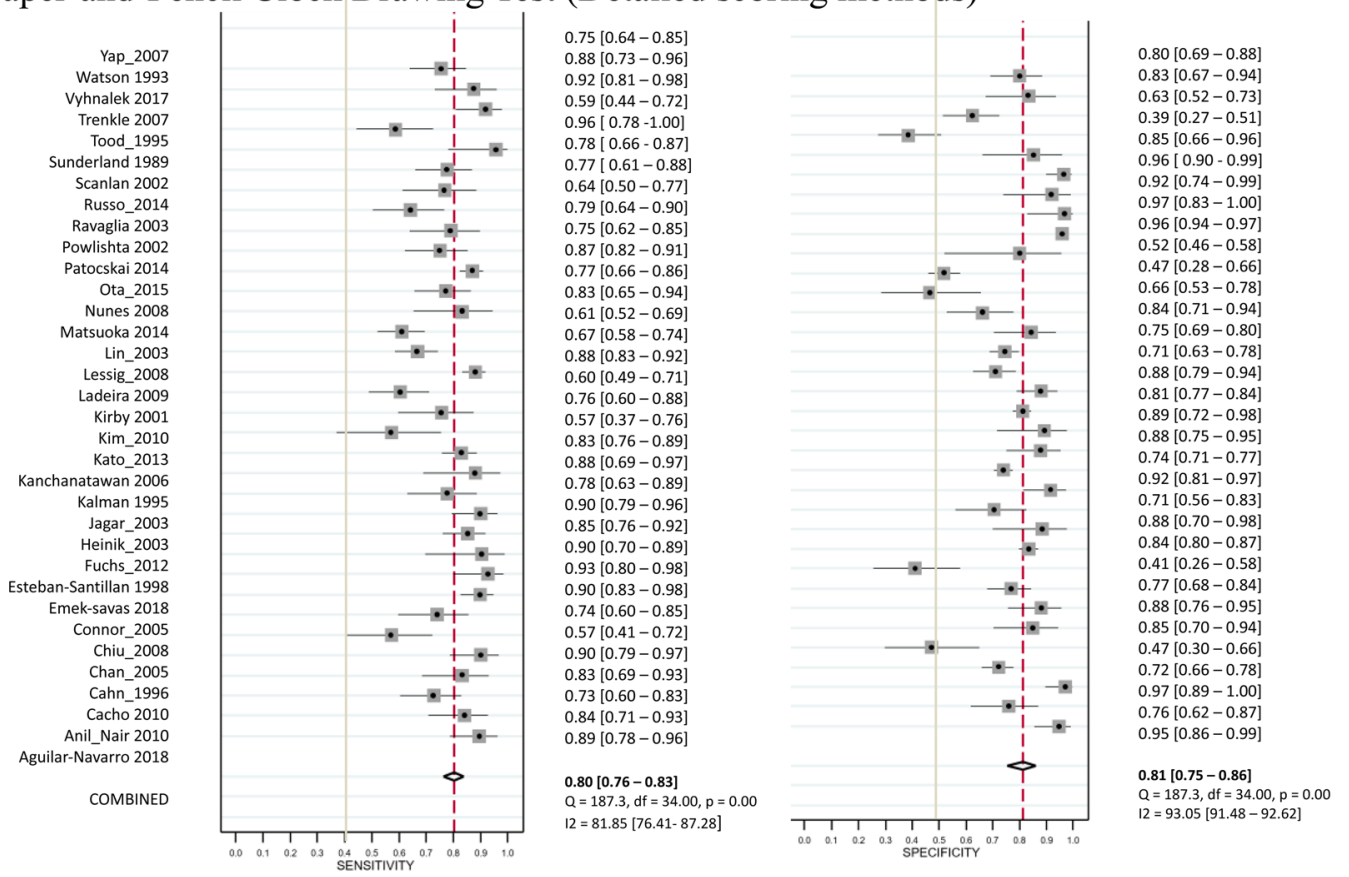

Fig. 3 Studies of Digital and Paper-and-Pencil Clock Drawing Tests for the Screening of Dementia 


\section{Performance of Other Types of Digital and Paper-and-pencil Drawing Tests}

In the screening of MCI, one study used digital ROCF (sensitivity: 0.76, specificity: 0.86, AUC: $85 \%$ ) and one study used paper-and-pencil ROCF (sensitivity: 0.59, specificity: 0.96, AUC: $77 \%$ ). In the screening of dementia, two studies used digital pentagon drawing test. The pooled sensitivity and specificity were $0.79(95 \%$ $\mathrm{CI}=0.74$ to 0.85$)$ and $0.74(95 \% \mathrm{CI}=0.68$ to 0.78$)$, respectively (Table $2 b$ ). Four studies used paper-andpencil pentagon drawing test. The pooled sensitivity and specificity were $0.85(95 \% \mathrm{CI}=0.70$ to 0.94$)$ and $73 \%$ $(95 \% \mathrm{CI}=0.52$ to 0.87$)$, respectively.

\section{Sensitivity Analyses}

Sensitivity analysis showed that the performance of different brief scoring method and detailed scoring method of paper-and-pencil CDT were comparable (Supplementary Table 5).

\section{Discussion}

This systematic review and meta-analysis included 90 studies and compared different types of digital drawing tests and paper-and-pencil drawing tests. The CDT is the most commonly used drawing test. In the screening of MCI, the digital CDT demonstrated better diagnostic performance than the paper-and-pencil CDT. Comparable performance was shown between the digital and paper-and-pencil CDT in the screeing of dementia. The diagnostic performance of other types of digital drawing tests and their paper-andpencil formats was also comparable. Therefore, digital drawing tests can used as an alternative tool for the screening of MCI and dementia.

There are similarities between the digital CDT and paper-andpencil CDT. Both methods require participants to draw the clock face as well as the hands of the clock that point to a specific time. The digital CDT uses a digital pen to draw on a tablet instead of drawing on a paper. Previous meta-analyses showed the diagnostic performance of paper-and-pencil CDT is fair in the screening of MCI, no matter the complexity of the scoring system (Ehreke et al.,

Table 2 Sensitivity and Specificity of Other types of Digital and Paper-and-Pencil Drawing Tests

\begin{tabular}{|c|c|c|c|c|c|c|c|}
\hline $\begin{array}{l}\text { Types of Drawing } \\
\text { Tests }\end{array}$ & No. of study & $\begin{array}{l}\text { Sensitivity } \\
(95 \% \mathrm{CI})\end{array}$ & $\begin{array}{l}\text { Specificity } \\
95 \% \text { CI) }\end{array}$ & $\mathrm{LR}+$ & LR- & DOR & AUC \\
\hline \multicolumn{8}{|l|}{ a. MCI } \\
\hline \multicolumn{8}{|l|}{ Digital Tests } \\
\hline $\begin{array}{l}\text { - Pentagon Draw- } \\
\text { ing }\end{array}$ & 1 & $0.71(0.44-0.99)$ & $0.86(0.66-1.00)$ & - & - & - & - \\
\hline$-\mathrm{ROCF}$ & 1 & 0.76 & 0.86 & - & - & - & $85 \%$ \\
\hline - Spiral Drawing & 1 & $1.00(0.72-1.00)$ & $1.00(0.97-1.00)$ & - & - & - & - \\
\hline - House Drawing & 1 & $0.85(0.61-1.00)$ & $0.94(0.79-1.00)$ & - & - & - & - \\
\hline \multicolumn{8}{|l|}{$\begin{array}{l}\text { Paper-and-pencil } \\
\text { Tests }\end{array}$} \\
\hline - Cube Drawing & 1 & 0.66 & 0.53 & - & - & - & \\
\hline - ROCF Drawing & 1 & 0.59 & 0.96 & - & - & - & $77 \%$ \\
\hline \multicolumn{8}{|l|}{ b. Dementia } \\
\hline \multicolumn{8}{|l|}{ Digital Tests } \\
\hline $\begin{array}{l}\text { - Pentagon Draw- } \\
\text { ing }\end{array}$ & 2 & $0.79(0.74-0.85)$ & $0.74(0.68-0.78)$ & $8.08(0.42-156)$ & $0.22(0.09-0.54)$ & $41.1(1.07-157)$ & - \\
\hline - Tree Drawing & 2 & $0.88(0.81-0.93)$ & $0.78(0.68-0.86)$ & $3.63(2.07-6.38)$ & $0.17(0.10-0.27)$ & $22.7(10.8-47.9)$ & - \\
\hline$-\mathrm{ROCF}$ & 1 & 0.82 & 0.91 & - & - & - & $93 \%$ \\
\hline - House Drawing & 1 & $0.81(0.69-0.94)$ & $1.00(0.94-1.00)$ & - & - & - & - \\
\hline - Spiral Drawing & 1 & $0.85(0.73-0.97)$ & $1.00(0.94-1.00)$ & - & - & - & - \\
\hline \multicolumn{8}{|l|}{$\begin{array}{l}\text { Paper-and-pencil } \\
\text { Tests }\end{array}$} \\
\hline $\begin{array}{l}\text { - Pentagon Draw- } \\
\text { ing }\end{array}$ & 4 & $0.85(0.70-0.94)$ & $0.73(0.52-0.87)$ & $3.21(1.82-5.66)$ & $0.20(0.11-0.36)$ & $16.1(9.92-26.3)$ & $\begin{array}{l}87 \%(84 \%- \\
90 \%)\end{array}$ \\
\hline - Cube Drawing & 1 & 0.74 & 0.63 & - & - & - & -- \\
\hline
\end{tabular}

$M C I$ Mild Cognitive Impairment, $R O C F$ Rey-Osterrieth complex figure, $L R+$ Positive likelihood ratio, $L R$ - Negative likelihood ration, $D O R$ Diagnostic odds ratio, AUC Area under the curve, $C I$ Confidence interval 
2010; Pinto \& Peters, 2009; Tsoi et al., 2017). This study showed similar results, however, the digital CDT showed better diagnostic performance than paper-and-pencil CDT in the screening of MCI. It may be due to the fact that deterioration of cognitive abilities such as executive function and visuospatial abilities found in patients with $\mathrm{MCI}$ are not yet clearly reflected in the final product of the paper drawing. However, decline in cognitive functions may be reflected in the drawing process captured in digital drawing tests (Müller et al., 2017; Garre-Olmo et al., 2017). Among different drawing characteristics, drawing time, pressure acceleration, and velocity are shown to be the behaviour markers for the discrimination of MCI from healthy aging (Garre-Olmo et al., 2017). Müller et al. (2019) further found that drawing time and velocity, such as time-in-air, total time strokes per minute are more sensitive maker than drawing pressure. Müller et al. (2017) suggested that time-in-air was a more sensitive marker than other time factors such as time-on-surface and total time (Muller et al., 2017). Additionally, the digital systems can automatically divide the drawing surface into different segments and sub-regions, and then analyze the strokes and angular differences of the drawing in the calculation of the final score (Davis et al., 2014; Shigemoria, et al., 2015). This combination of visual features and behavioural data can contribute to the identification of patients and enhance the accuracy of the digital CDT (Muller et al., 2019). Therefore, the use of digital CDT can improve the sensitivity and specificity in the screening of MCI. Past work suggests that machine-learning methods can enhance the ability to produce accurate predictive models of the drawing tests to classify $\mathrm{MCI}$ and dementia when the models trained on a large amount of data (Davis et al., 2014, Souillard-Mandar et al., 2016; Muller et al., 2017, 2019). Besides digital CDT, some other digital drawing tests have been suggested in the literature. The digital pentagon drawing test (Garre-Olmo et al., 2017; Tsoi et al., 2018) and digital ROCF (Cheah et al., 2019; Kokubo et al., 2018) are adapted from paper-and-pencil versions. The digital tree drawing test and digital house drawing test are adopted new approaches (Robens et al., 2019; Garre-Olmo et al., 2017). The digital tree drawing test and digital house drawing test are free-hand drawing tests which do not require the participants to draw any specific features. Thus, only drawing behaviour are used in the classification of disease.

A comprehensive evaluation of different types of drawing tests for the screening of MCI and dementia is the strength of this study. However, this study has some limitations. First, the comparisons in this study are not head-to-head comparisons, and patients' engagement and performance on the drawing test is a confounding factor to result interpretation. There is a study of head-to-head comparison between a digital drawing test and a paper-and-pencil drawing test, which showed that digital CDT had a higher diagnostic accuracy than paper-andpencil CDT (Müller et al., 2017). Second, the number of studies to compare diagnostic performance of drawing tests are limited. The benefits of digital drawing tests may be stronger if we can include more studies in this meta-analysis.

\section{Conclusions}

The current study revealed that digital CDT can enhance the identification of deficits in the screening of MCI. Digital and paper-and-pencil CDT have a comparable performance in the screening of dementia. Other types of drawing tests in digital formats showed comparable to paper-in-pencil formats. Therefore, digital drawing tests can be a potential tool to use as an alternative for the screening of MCI and dementia.

Supplementary Information The online version contains supplementary material available at https://doi.org/10.1007/s11065-021-09523-2.

Acknowledgements We thank the INS Global Engagement Committee Research Editing and Consulting Program (RECP) and Dr. Sarah L. Garcia-Beaumier, consultant of the RECP, for help in manuscript editing.

Funding Source This is a self-initiated study. No funding was received.

\section{Declarations}

Ethical Approval This study was a systematic review and meta-analysis and ethical approval was not required.

Conflict of Interests None.

Open Access This article is licensed under a Creative Commons Attribution 4.0 International License, which permits use, sharing, adaptation, distribution and reproduction in any medium or format, as long as you give appropriate credit to the original author(s) and the source, provide a link to the Creative Commons licence, and indicate if changes were made. The images or other third party material in this article are included in the article's Creative Commons licence, unless indicated otherwise in a credit line to the material. If material is not included in the article's Creative Commons licence and your intended use is not permitted by statutory regulation or exceeds the permitted use, you will need to obtain permission directly from the copyright holder. To view a copy of this licence, visit http://creativecommons.org/licenses/by/4.0/.

\section{References}

Albert, M. S., DeKosky, S. T., Dickson, D., Dubois, B., Feldman, H. H., Fox, N. C., \& Phelps, C. H. (2011). The diagnosis of mild cognitive impairment due to Alzheimer's disease: Recommendations from the national institute on Aging-Alzheimer's association workgroups on diagnostic guidelines for Alzheimer's disease. Alzheimer's \& Dementia, 7, 270-279.

American Psychiatric Association. (2013). Diagnostic and statistical manual of mental disorders, 4th ed, revised. (DSM-IV-TR). Washington, DC: American Psychiatric Association.

Aprahamian, I., Martinelli, J. E., Neri, A. L., \& Yassuda, M. S. (2009). The Clock Drawing Test A review of its accuracy in screening for dementia. Dement Neuropsychol, 3(2), 74-81.

Bossuyt, P. M., Reitsma, J. B., Bruns, D. E., Gatsonis, C. A., Glasziou, P. P., Irwig, L. M., \& Standards for Reporting of Diagnostic Accuracy. (2003). The STARD statement for reporting studies 
of diagnostic accuracy: Explanation and elaboration. Annals of Internal Medicine, 138, W1-12.

Breton, A., Casey, D., \& Arnaoutoglou, N. A. (2019). Cognitive tests for the detection of mild cognitive impairment (MCI), the prodromal stage of dementia: Meta-analysis of diagnostic accuracy studies. International Journal of Geriatric Psychiatry, 34(2), 233-242.

Chan, J. Y. C., Kwong, J. S. W., Wong, A., Kwok, T. C. Y., \& Tsoi, K. K. F. (2018). Comparison of Computerized and Paperand-Pencil Memory Tests in Detection of Mild Cognitive Impairment and Dementia: A Systematic Review and Metaanalysis of Diagnostic Studies. Journal of the American Medical Directors Association, 19(9), 748-756.e5.

Cheah, W. T., Chang, W. D., Hwang, J. J., Hong, S. Y., Fu, L. C., \& Chang, Y. L. (2019). A Screening System for Mild Cognitive Impairment Based on Neuropsychological Drawing Test and Neural Network. 2019 IEEE International Conference on Systems, Man and Cybernetics (SMC), Bari, Italy.

Cormack, F., Aarsland, D., Ballard, C., \& Tovée, M. J. (2004). Pentagon drawing and neuropsychological performance in Dementia with Lewy Bodies, Alzheimer's disease, Parkinson's disease and Parkinson's disease with dementia. International Journal of Geriatric Psychiatry, 19(4), 371-377.

Davis, R., Libon, D. J., Au, R., Pitman, D., \& Penney, D. L. (2014). Think: Inferring Cognitive Status from Subtle Behaviors. Proceedings of the AAAI Conference on Artificial Intelligence, 2014, 2898-2905.

Deeks, J. J., Macaskill, P., \& Irwig, L. (2005). The performance of tests of publication bias and other sample size effects in systematic reviews of diagnostic test accuracy was assessed. Journal of Clinical Epidemiology, 58(9), 882-893.

DerSimonian, R., \& Laird, N. (1986). Meta-analysis in clinical trials. Controlled Clinical Trials, 7, 177-188.

Ehreke, L., Luppa, M., Konig, H. H., \& Riedel-Heller, S. G. (2010). Is the Clock Drawing Test a screening tool for the diagnosis of mild cognitive impairment? A Systematic Review. International Psychogeriatrics, 22(1), 56-63.

Faundez-Zanuy, M., Sesa-Nogueras, E., Roure-Alcobé, J., Garré-Olmo, J., Lopez-de-Ipiña, K., \& Solé-Casals, J. (2013). Online Drawings for Dementia Diagnose: In-Air and Pressure Information Analysis. In: Roa Romero L. (eds) XIII Mediterranean Conference on Medical and Biological Engineering and Computing. IFMBE Proceedings, vol 41. Springer, Cham.

Garre-Olmno, J., Faundez-Zanuy, M., Lopez-de-Ipina, K., Calvo-Perxas, L., Turro-Garriga1, O. (2017). Kinematic and pressure features of handwriting and drawing: preliminary results between patients with mild cognitive impairment. Alzheimer Disease and Healthy Controls Current Alzheimer Research, 14(9), 960968.

Glas, A. S., Lijmer, J. G., Prins, M. H., Bonsel, G. J., \& Bossuyt, P. M. M. (2003). The diagnostic odds ratio: A single indicator of test performance. Journal of Clinical Epidemiology, 56(11), 1129-1135.

Heymann, P., Gienger, R., Hett, A., Müller, S., Laske, C., Robens, S., \& Elbing, U. (2018). Early Detection of Alzheimer's Disease Based on the Patient's Creative Drawing Process: First Results With a Novel Neuropsychological Testing Method. Journal of Alzheimer's Disease, 63(2), 675-687.

Kokubo, N., Yoko, Y., Saitoh, Y., Murata, M., Maruo, K., Takebayashi, Y., \& Horikoshi, M. (2018). A new device-aided cognitive function test, User eXperience-Trail Making Test (UX-TMT), sensitively detects neuropsychological performance in patients with dementia and Parkinson's disease. BMC Psychiatry, 5;18(1):220.

Leeflang, M. M., Deeks, J. J., Gatsonis, C., Bossuyt, P. M. M., \& Cochrane Diagnostic Test Accuracy Working Group. (2008). Systematic reviews of diagnostic test accuracy. Annals of Internal Medicine, 149, 889-897.
Macaskill, P., Gatsonis, C., Deeks, J., Harbord, R., \& Takwoingi, Y. (2010). Chapter 10: analysing and presenting results. In: Deeks, J.J., Bossuyt, P.M. \& Gatsonis, C. (editors). Cochrane handbook for systematic reviews of diagnostic test accuracy version 1.0. the cochrane collaboration. Available from: http://srdta.cochrane.org/

McKhann, G., Drachman, D., Folstein, M., Katzman, R., Price, D., \& Stadlan, E. M. (1984). Clinical diagnosis of Alzheimer's disease: Report of the NINCDS-ADRDA work group under the auspices of department of health and human services task force on alzheimer's disease. Neurology, 34(7), 939-944.

Moher, D., Liberati, A., Tetzlaff, J., Altman, D. G., \& PRISMA Group. (2009). Preferred reporting items for systematic reviews and metaanalyses: The PRISMA statement. Annals of Internal Medicine, $151,264-269$.

Morris, J. C. (1993). The clinical dementia rating (CDR): Current version and scoring rules. Neurology, 43(11), 2412-2414.

Müller, S., Herde, L., Preische, O., Zeller, A., Heymann, P., Robens, S., \& Laske, C. (2019). Diagnostic value of digital clock drawing test in comparison with CERAD neuropsychological battery total score for discrimination of patients in the early course of Alzheimer's disease from healthy individuals. Science and Reports, 9(1), 3543.

Müller, S., Preische, O., Heymann, P., Elbing, U., \& Laske, C. (2017). Increased diagnostic accuracy of digital vs. conventional clock drawing test for discrimination of patients in the early course of alzheimer's disease from cognitively healthy individuals. Frontiers in Aging Neuroscience, 11(9), 101.

Nasreddine, Z. S., Phillips, N. A., Bédirian, V., Charbonneau, S., Whitehead, V., Collin, I., \& Chertkow, H. (2005). The Montreal Cognitive Assessment, MoCA: A brief screening tool for mild cognitive impairment. Journal of the American Geriatrics Society, 53(4), 695-699.

Ota, K., Murayama, N., Kasanuki, K., Kondo, D., Fujishiro, H., Arai, H., \& Iseki, E. (2015). Visuoperceptual assessments for differentiating dementia with lewy bodies and alzheimer's disease: illusory contours and other neuropsychological examinations. Archives of Clinical Neuropsychology, 30(3), 256-263.

Park, J. K., Jeong, E. H., \& Seomun, G. A. (2018). The clock drawing test: A systematic review and meta-analysis of diagnostic accuracy. Journal of Advanced Nursing Actions, 74(12), 2742-2754.

Pal, A., Biswas, A., Pandit, A., Roy, A., Guin, D., Gangopadhyay, G., \& Senapati, A. K. (2016). Study of visuospatial skill in patients with dementia. Annals of Indian Academy of Neurology, 19, 83-88.

Petersen, R. C., Smith, G. E., Waring, S. C., Ivnik, R. J., Tangalos, E. G., \& Kokmen, E. (1999). Mild cognitive impairment: Clinical characterization and outcome. Archives of Neurology, 56, 303-308.

Pinto, E., \& Peters, R. (2009). Literature review of the Clock Drawing Test as a tool for cognitive screening. Dementia and Geriatric Cognitive Disorders, 27, 201-213.

Prince, M., Bryce, R., \& Ferri. C. (2011) The benefits of early diagnosis and intervention. Alzheimer's Disease International World Alzheimer Report 2011, available at https://www.alz.co.uk/resea rch/world-report-2011 assessed at 18 April 2020.

Reitsma, J. B., Glas, A. S., Rutjes, A. W., Scholten, R. J. P. P., Bossuyt, P. M., \& Zwinderman, A. H. (2005). Bivariate analysis of sensitivity and specificity produces informative summary measures in diagnostic reviews. Journal of Clinical Epidemiology, 58, 982-990.

Robens, S., Heymann, P., Gienger, R., Hett, A., Müller, A., Laske, C., \& Elbing, U. (2019). The Digital Tree Drawing Test for Screening of Early Dementia: An Explorative Study Comparing Healthy Controls, Patients with Mild Cognitive Impairment, and Patients with Early Dementia of the Alzheimer Type. Journal of Alzheimer's Disease, 68(4), 1561-1574. 
Rutter, C. M., \& Gatsonis, C. A. (2001). A hierarchical regression approach to meta-analysis of diagnostic test accuracy evaluations. Statistics in Medicine, 20, 2865-2884.

Shigemoria, T., Harbi, Z., Kawanaka, H., Hicks, Y., Setchi, R., Takase, H., \& Tsuruoka, S. (2015). Feature Extraction Method for Clock Drawing Test. Procedia Computer Science, 60, 1707-1714.

Shulman, K. I., Shedletsky, R., \& Silver, I. L. (1986). The challenge of time: Clock-drawing and cognitive function in the elderly. International Journal of Geriatric Psychiatry, 1, 135-140.

Souillard-Mandar, W., Davis, R., Rudin, C., Au, R., Libon, D. J., Swenson, R., \& Penney, D. L. (2016). Learning Classification Models of Cognitive Conditions from Subtle Behaviors in the Digital Clock Drawing Test. Mach Learn Actions, 102(3), 393-441.

Sunderland, T., Hill J. L., Mellow, A. M., Lawlor, B. A., Gundersheimer, J., Newhouse, P.A., \& Grafman, J, H. (1989). Clock drawing in alzheimer's disease. A novel measure of dementia severity. Journal of the American Geriatrics Society, 37(8), $725-729$.

Traykov, L., Raoux, N., Latour, F., Gallo, L., Hanon, O., Baudic, S., et al. (2007). Executive Functions Deficit in Mild Cognitive Impairment. Cognitive and Behavioral Neurology, 20(4), 219-224.

Tsoi, K. K. F., Chan, J. Y. C., Hirai, H. W., Wong, S. Y. S., \& Kwok, T. C. Y. (2015). Cognitive tests to detect dementia. A systematic review and meta-analysis. JAMA Internal Medicine, 175, $1450-1458$.
Tsoi, K. K. F., Chan, J. Y. C., Hirai, H. W., Wong, A., Mok, V. C. T., Lam, L. C. W., \& Wong, S. Y. S. (2017). Recall Tests Are Effective to Detect Mild Cognitive Impairment: A Systematic Review and Meta-analysis of 108 Diagnostic Studies. Journal of the American Medical Directors Association, 18(9), 807.e17-807.e29.

Tsoi, K. K. F., Lam, M. W. Y., Chu, C., Wong, M. P. F., \& Meng, H. (2018). Machine Learning on Digital Drawing Date For Preliminary Dementia Screening. Alzheimer's and Dementia, 14(7), 196. https://doi.org/10.1016/j.jalz.2018.06.2039

Whiting, P. F., Rutjes, A. W. S., Westwood, M. E., Mallett, S., Deeks, J. J., Reitsma, J. B., \& QUADAS-2 Group. (2011). QUADAS-2: A revised tool for the quality assessment of diagnostic accuracy studies. Annals of Internal Medicine, 155, 529-536.

Winblad, B., Palmer, K., Kivipelto, M., Jelic, V., Fratiglioni, L., Wahlund, L. O., \& Petersen, R. C. (2004). Mild cognitive impairment - Beyond controversies, towards a consensus: Report of the international working group on mild cognitive impairment. Journal of Internal Medicine, 256, 240-246.

World Health Organization. (2019). Dementia. Retrieved on January 12 2021. Retrieval from: https://www.who.int/news-room/factsheets/detail/dementia

Publisher's Note Springer Nature remains neutral with regard to jurisdictional claims in published maps and institutional affiliations. 